[Aus dem Institut für Infectionskrankheiten zu Berlin.]

\title{
Wirkung der Influenzabacillen auf das Centralnerven- System. ${ }^{1}$ \\ Von
}

Dr. A. Cantani jun.

\begin{abstract}
Durch die klassischen Untersuchungen von R. Pfeiffer ist das Dunkel, welches über das Wesen des Influenzaprocesses berrschte, in mehr als einer Hinsicht gehoben und aufgeklärt. Die Entdeckung des Influenzabacillus ist in dieser Hinsicht von grosser Bedeutung gewesen, denn dadurch war die Möglichkeit gegeben, einen näheren Einblick über das Zustandekommen des Krankheitsbildes zu gewinnen. Pfeiffer konnte den Nachweis erbringen, dass die Influenzabakterien lediglich in den Respirationswegen localisirt sind und dass von hier aus alle beobachteten Erscheinungen ihren Ursprung nehmen mussten. Um nun den täglich zu beobachtenden schweren allgemeinen Leiden Rechnung zu tragen, griff Pfeiffer zu der Hypothese der Giftproduction der Influenzabacillen und nahm an, dass die Influenzabakterien toxische Producte produciren müssen, welche in erster Linie auf die cerebralen Centren einwirken. Diese Ansicht von Pfeiffer ist heute wohl allgemein anerkannt, nachdem man darauf aufmerksam geworden ist, dass die Influenza nicht nur Erscheinungen im Respirationstractus, sondern auch von Seiten des Centralnervensystems auslöst. In einem Theile dieser nervösen Influenzafälle hat man die Influenzabakterien auch in den cerebralen Wegen nachweisen können. So macht Pribram auf schwere Gehirnerscheinungen aufmerksam, für die er das Einwandern von Influenzabacillen in die Gehirnhäute ver-

Eingegangen am 20. August 1896.
\end{abstract}


antwortlich machen will. In fünf Fällen unter schweren Erscheinungen zu Tode führender Influenza fand $\mathrm{Pfuhl}$ in den Blutextravasaten und der Ventrikelfüssigkeit kleine Bacillen, die er für identisch erachtet mit den Pfeiffer'schen Influenzabacillen. Auf Glycerinagar gelang es ihm dieselben nur in erster Generation zum Wachsthum zu bringen.

Auf der dritten Versammlung der Deutschen otologischen Gesellschaft in Bonn machte Hartmann die Mittheilung, dass bei Otitis media der Säuglinge sehr häufig Influenzabacillen gefunden werden. Auf diese Thatsache hatte schon früher auch Kossel hingewiesen.

In anderen klinisch als Influenza imponirenden Fällen ist allerdings der Nachweis von Influenzabakterien nicht gelungen, es kann aber auch in der Unsicherheit der Untersuchung begründet sein. Leyden beschreibt zunächst einen Fall, wo sich uach Kopfschmerzen, Erbrechen, Mattigkeit, die er bei der herrschenden Epidemie als Influenzaerscheinungen anzusehen sich berechtigt glaubt, in der Folge Oedem und Albuminurie und einige Wochen später Druckempfindlichkeit, Hyper- und Hypästhesie, quantitative Herabsetzung der Erregbarkeit in einzelnen Muskeln und Nerven einstellte, in anderen theilweise Entartungsreaction. Der Fall kam nach schwerem Verlauf in einigen Monaten wieder zur Genesung.

Der zweite Fall betrifft eine Erkrankung an Landry'scher Paralyse mit Polyneuritis nach Influenza. Hirschmann beschreibt vier Fälle, wo sich in der Reconvalescenz nach Influenza eine doppelseitige Entzündung der Sehnerven an der Eintrittsstelle mit Uebergreifen auf die umgebende Retina ausbildete.

Lemecke theilt mit, dass unter 64 Kranken, die in Folge von Influenza am $\mathrm{Ohr}$ litten, bei 11 das Antrum mastoideum eröffnet werden musste. Bei 4 davon handelt es sich um Caries und Nekrose. Fr vergleicht diese Erkrankung nach Influenza mit-dem Knochenprocesse bei acuter Osteomyelitis. Seine Ansicht geht dahin, dass die Influenzaotitis nicht selten Primärerkrankungen der Knochen hervorrufen.

Voges theilt einen interessanten hierher gehörigen Fall mit, in welchem der Patient 14 Tage lang an intensivem Kopfsehmerz und Fieber, begleitet von wüthenden anfallsweise auftretenden Schmerzen in der Gegend der rechten Hygmorshöhle und des rechten Ohres, litt. Allmählich verbreitete sich über den ganzen Körper eine sehr stark ausgeprägte Hyperästhesie, so dass die leiseste Berührung ein schmerzhaftes Zusammenzucken des ganzen Körpers hervorruft. Dieser fieberhafte rein nervöse Fall ist besonders deswegen interessant, weil am 12. Tage nach der Erkrankung, als zum ersten Mal einige Ballen eines grün-gelben Lungensputums ausgeworfen wurden, in demselben nahezu eine Reincultur von Influenzabacillen gefunden wurde. 
Diese rein nervösen Fälle von Influenza können ebensowohl wie die bei der bronchitischen Form der Influenza zu beobachtenden Erscheinungen von Seiten des Centralnervensystems, nur mit Hülfe der Pfeiffer'schen Hypothese über eine Giftwirkung der Influenzabakterien eine befriedigende Erklärung finden. Um so interessanter müsste es sein, diese Fragen an Thieren experimentell zu studiren, um einmal den Infectionsmodus kennen zu lernen, andererseits aber auch die Natur des Toxins und die Art der Giftwirkung zu ermitteln. Pfeiffer betont indess, dass spontan eine Uebertragung der Influenza auf irgend eine Thierspecies nicht beobachtet worden ist, er selbst hat dann zahlreiche Versuche angestellt mit Mäusen, Ratten, Meerschweinchen, Kaninchen, Schweinen, Katzen, Hunden und Affen. Nur beim Affen ist ihm die Erzeugung eines infectiösen Processes gelungen. Bei anderen Thieren konnte er nur durch Vergiftung den Tod herbeiführen. Das Influenzagift bedingt bei Kaninchen in die Blutbahn gebracht Dispnoë und lälımungsartige Schwäche der Muskulatur. Meerschweinchen und Mäuse vertrugen relativ enorme Mengen der Influeuzabacillen.

Ausser Pfeiffer, welcher wohl die zahlreichsten Versuche über Thierpathogenität der Influenzabakterien angestellt hat, finde ich in der Litteratur, soweit sie mir zugänglich ist, nur in einer Arbeit von Voges Versuche an Kaninchen und Mäusen. Derselbe fasst auch die durch Infection der Mäuse und Kaninchen erzeugte Erkrankung mit Influenzabacillen als eine reine Intoxication auf, bei der eine Infection ausgeschlossen werden muss.

Die beim Menschen beobachteten schweren nervösen Symptome schienen mir darauf hinzudeuten, einen Hauptangriffspunkt der Influenzabakterien im Gehirn suchen zu sollen, in der Verfolgung der Pfeifferschen Hypothese, dass das Influenzagift ein Nervengift ist. Aus diesem Grunde glaubte ich mich für berechtigt zu halten, auch im Thierexperiment die Influenzabakterien direct an den Ort ihrer Wirkungsstätte appliciren zu sollen, um so den directesten Einfluss derselben auf das Centralnervensystem studiren zu können. Andererseits durfte man auch die Erwartung hegen, dass der Angriffspunkt der Influenzabakterien auf den Thierkörper gerade vom Gehirn aus ein besonders günstiger sei, nachdem wir gesehen haben, dass andere Infectionsmodi, wie sie von früheren Autoren benutzt wurden, wenig erfolgreich waren.

Trepanation und Injectionen von fremdartigen Stoffen bilden immer einen schweren Eingriff in die vitalen Functionen des thierischen Organismus, es schien daher geboten, bevor ich zu den Versuchen Influenzabakterien in das Gehirn einzuspritzen, überging, Controlversuche anzustellen, um die Wirkung des operativen Eingriffes an sich kennen zu 
lernen. Zu dem Zwecke habe ich ausgewachsenen Kaninchen nach gelungener Trepanation verschiedene Mengen sterilen destillirten Wassers und Bouillon in die Gehirnsubstanz eingespritzt, anderen Thieren habe ich wiederum mit sterìler Canüle das Gehirn nach verschiedenen Richtungen hin arg beschädigt, habe mich aber überzeugen können, dass, wenn der Eingriff in den vorderen Theil des Gehirns fällt und die zu injicirende Flüssigkeitsmenge keine allzu hohe $(d . h .1 / 2 \mathrm{~cm}$ nicht übersteigende) war, das Thier in seinem Wohlbefinden nicht wesentlich gestört wurde; selbst wenu gleich nach der Operation Krämpfe auftraten, verschwanden diese bald wieder und das Thier war am nächsten Tage wieder völlig munter.

Es war nun die Möglichkeit gegeben, dass durch die Operation die Thiere in der Weise geschädigt waren, dass sie einem Angriffe pathogener Bakterien gegenüber in ihrer Widerstandsfähigkeit herabgesetzt waren, um auch diese Verhältnisse zu prüfen, habe ich Kaninchen trepanirt und gleichzeitig intraperitoneal mässig virulente Fränkel'sche Diplokokken injicirt, während die Controlthiere nur der Intraperitonealinfection ausgesetzt wurden.

Kaninchen 138. $1680^{\mathrm{grm}}$. Ins Gehirn $1 / 2 \mathrm{ecm}$ sterilisirtes Wasser. In die Bauchhöhle 1 Oese Condenswasser einer Fränkel'schen Diplokokkencultur. Erfolg: Thier bleibt am Leben.

Kaninchen 139. $1690^{\mathrm{grm}}$. In die Bauchhöhle 1 Oese Condenswasser desgl. Erfolg: Bleibt am Leben.

Kaninchen 129. $1580^{\mathrm{grm}}$. Ins Gehirn $1 / 2{ }^{\mathrm{ccm}}$ sterilisirtes Wasser. In die Bauchhöhle 3 Oesen derselben Aufschwemmung. Erfolg: Das Thier stirbt nach 3 Tagen mit Septicämie.

Kaninchen 130. $1630 \mathrm{grm}$. In die Bauchhöhle 3 Oesen desgl. Erfolg: Stirbt nach 62 Stunden mit Septicämie.

Aus diesen Versuchen geht hervor, dass der operative Eingriff an sich keine Schädigung hervorzurufen braucht; eine Bestätigung dieses Ergebnisses finde ich auch in den zahlreichen Versuchen, in denen nach Injectionen von nicht tödtlichen Mengen pathogener Bakterien die trepanirten Kaninchen, ohne irgend welche Krankheitssymptome zu zeigen, am Leben geblieben sind. Ich kann daher dem Trauma böchstens nur eine untergeordnete Rolle vindiciren.

Nach Festlegung dieser Thatsache können wir zu Versuchen mit Influenzabakterien übergehen, indem wir uns bewusst bleiben, dass die in diesen Experimenten auftretenden Erscheinungen nur als Ausdruck der Bakterieninfection angesehen werden müssen. 
Ich schicke voraus, dass es mir gelungen ist, die Kaninchen schou durch Injectionen von relativ geringen Dosen lebender Influenzabakterien zu tödten; die während der Krankheit zu beobachtenden Symptome sind in Kürze folgende:

Nachdem sich die Thiere von dem operativen Eingriffe erholt haben, sind sie in der Regel äusserlich völlig gesund, erst nach 8 bis 10 Stunden beobachten wir die ersten Krankheitsmerkmale; diese äussern sich als Temperatursteigerung; diese kann schon nach 6 Stunden ihr Maximum erreicht haben, je nach der Grösse der injicirten Dosis, ebenso häufig kann die Akme bis $42^{\circ} \mathrm{C}$. auch erst nach 8 bis 12 Stunden eintreten, dann setzt eine Remission ein, welche in wenigen Stunden progressiv die Norm überschreitet und zu subnormalen Wärmegraden führt, bis das Thier 18 bis 36 Stunden nach der Infection eingeht. War die Dosis nicht tödtlich, so geht die Temperatur nach mehr oder weniger langem Anhalten des fieberhaften Zustandes im Verlauf von 28 Stunden zur Norm zurück, um normal zu bleiben.

Einhergehend mit der fieberhaften Steigerung der Eigenwärme, beobachtet man eine stetig zunehmende, auf dem Höhepunkt sehr ausgesprochene Dyspnö̈; gleichzeitig setzt eine Paralyse ein, die mit den Hinterbeinen beginnend sich von hinten nach vorne zu über den ganzen Körper verbreitet, daher vermögen die Thiere sich im Anfang nur noch mit den Vorderbeinen fortzubewegen, indem sie den Hinterkörper nachschleifen; bei Berührung und spontanen Bewegungsversuchen werden äusserst stürmische clonische Krämpfe ausgelöst. Allmählich erlahmt auch die Vorderextremität; die Thiere liegen willenlos auf der Seite und vermügen bald auch den Kopf und die Ohren nicht mehr zu heben; dieser Scheintodtzustand hält noch stundenlang an, das Leben ist nur gekennzeichnet durch die Dyspnoë und herabgesetzte Herzthätigkeit. Hin und wieder geben die Thiere einen langanhaltenden Schrei von sich. In diesem entsetzlich elenden Zustande gehen die Thiere endlich ein.

Nach Application von nicht tödtlicher Dosis beobachten wir den geschilderten Symptomencomplex in mehr oder weniger ausgesprochener Weise, es entwickelt sich alsdann häufig eine chronische Meningitis, welche in einzelnen Fällen noch letal enden kann, in anderen aber wiederum in Genesung übergeht. Bei Anwendung noch geringerer Dosen können die Symptome auf ein Minimum beschränkt sein.

Die minimal tödtliche Dosis der ins Gehirn eingebrachten lebenden Influenzabakterien ist schwankend und hängt von der jeweiligen Virulenz der Culturen ab. Mit mässig virulenten Culturen starben die Thiere mit $0.5^{\mathrm{mg}}$ einer 20 stündigen Blutagarcultur. Bei sehr virulenten Culturen genügten dagegen geradezu minimale Bruchtheile derselben, wie 2 Oesen 
der in $1^{\mathrm{cem}}$ Bouillon aufgeschwemmten Cultur, um das Thier in 24 Stunden zu tödten. In der nächsten Tabelle werden Versuche mit verschiedenen Mengen von aus verschiedener Quelle stammenden Culturen vorgeführt.

$$
\text { Cultur IB a (24 St.). }
$$

Kaninchen 12. $1920^{\mathrm{grm}}$. $1 / 2 \mathrm{~cm}$ Bouillon enthaltend $1 / 10$ Cultur. Erfolg: Stirbt nach ungefähr 20 Stunden. Aus dem Gehirn reine Culturen von Influenza.

Kaninchen 13. $1500^{\mathrm{grm}}$. $1 / 2{ }^{\mathrm{em}}$ Bouillon mit $1 / 20$ Cultur. Erfolg: Stirbt nach 30 Stunden. Aus dem Gehirn reine Culturen von IB.

Kaninchen 14. $1770^{\mathrm{grm}}$. $1 / 2{ }^{\mathrm{cem}}$ Bouillon mit $1 / 40$ Cultur. Erfolg: Leichte Dyspnoë und Paralyse der Hinterbeine. Bleibt am Leben.

\section{Cultur IB. K.}

Kaninchen 24. $1760 \mathrm{grm}$. $1 / 2 \mathrm{ccm}$ Bouillon mit 2 Oesen der in $1 \mathrm{~cm}$ Wasser verdünnten Cultur. Erfolg: Am nächsten Tage Parlyse der Hinterbeine, clonische Krämpfe, endlich totale Paralyse. Stirbt nach 24 Stunden. Aus dem Gehirn reine Culturen von IB.

Kaninohen 41. $1390^{\mathrm{grm}}$. 1/4 ${ }^{\mathrm{cem}}$ Bouillon mit 1 Oese desgl. Erfolg: Am nächsten Tage Dyspnoë, leichte Paralyse, Nackenzuckungen. Bleibt am Leben.

Cultur IB. M. 163. Auf mit Blut gemischtem Agar.

Kaninchen 212. $1670^{\mathrm{grm}}$. $1 / 2 \mathrm{cem}$ Bouillon enthaltend $4 \mathrm{mg}$ IB. Erfolg: Stirbt nach 20 Stunden. Reine Cultur aus dem Gehirn gezüchtet.

Kaninchen 213. $1750^{\mathrm{prm}}$. $1 / 2{ }^{\mathrm{ccm}}$ Bouillon mit $2 \mathrm{mg}$ IB. Erfolg: Am nächsten Tage Paralyse, Kopf wackelnd. Stirbt nach 30 Stunden. Reine Cultur.

Kaninchen 214. $1590^{\mathrm{grm}} .1 / 2{ }^{\mathrm{ccm}}$ Bouillon mit $11^{\mathrm{mg}}$ IB. Erfolg: Am nächsten Tage Paralyse, Dyspnoë. Stirbt nach ungefähr 30 Stunden. Reine Cultur.

Kaninchen 223. $1620^{\mathrm{krm}}$. $1 / 2{ }^{\mathrm{ecm}}$ Bouillon mit $1 / 2^{\mathrm{mg}}$ IB. Erfolg: Gestorben nach 24 Stunden.

Kaninchen 229. $1500^{\mathrm{grm}}$. $1 / \mathrm{e}^{\mathrm{ecm}}$ Bouillon mit $1 / 4 \mathrm{mg}$ IB. Erfolg: Am nächsten Tage leichte Paralyse. Bleibt am Leben.

Kaninehen 230. $1500 \mathrm{grm}$. $1 / 8{ }^{\mathrm{ecm}}$ Bouillon mit $1 / 8 \mathrm{mg}$ IB. Erfolg: Bleibt am Leben.

Aus den mitgetheilten Versuchen ergiebt sich, dass die Virulenz der einzelnen Influenzastämme eine schwankende ist; die mittlere Virulenz beträgt bei unserem Infectionsmodus indess etwa $1 / 2$ bis $1 \mathrm{mg}$ einer 24 stündigen Blutagareultur.

Bei den gestorbenen Thieren ergab der Obductionsbefund alle die allgemeinen Erscheinungen eines an einer starken Infection eingegangenen Thieres. Es fand sich sehr oft ein blutigseröses Exsudat in der Bauch- 
höhle, die Gefässe des Peritoneums waren stark angefüllt, die Milz oft vergrössert und hyperämisch, die Nieren sehr hyperämisch mit Merkmalen von einer acuten Nephritis, der Urin oft bluthaltig, die Nebennieren oft stark geröthet, die Leber immer sehr hyperämisch und in beginnender Verfettung. Die Lungen waren immer blutreich, im Pericard war oft eine ziemlich grosse Menge klarer durchsichtiger Flüssigkeit. Im Blute, im Peritonealexsudat und in allen Organen habe ich nie, weder mikroskopisch noch culturell, Influenzabakterien nachweisen können. An der Trepanationsstelle bemerkte man oft ein subcutanes gallartiges Oedem, welches zahlreiche Influenzabakterien enthielt, und hämorrhagische Flecken; die Meningen waren immer stark hyperämisch verdickt und getrübt; oft gab es hämorrhagische Ergüsse. Sie enthielten immer ein blutigseröses Exsudat. Das Gehirn zeigte sich makroskopisch stark hyperämisch, die Ventrikel enthielten ein oft eitriges Exsudat, in welchem Influenzabakterien mikroskopisch und culturell nachweisbar waren. Die Schnittfläche des Gehirns zeigte die graue Substanz etwas geröthet und von zahlreichen punktförmigen Blutungen durchsetzt. Mikroskopisch waren in der Gehirnsubstanz massenhaft Influenzabakterien vorhanden. In Schnitten sah man in der Gehirnsubstanz reichliche Infiltration von weissen Blutkörperchen und zahlreiche mehr oder weniger ausgedehnte Hämorrhagieen. (Acute Encephalitis.) Zahlreiche Influenzabakterien waren auch in Schnitten zu sehen, so dass eine Vermehrung derselben in der Gehirnsubstanz unzweifelhaft war. Die Bakterien scheinen sich durch die Lymphgefässe mit besonderer Vorliebe zu verbreiten, man konnte oft die Bahn eines Lymphgefässes an den darin enthaltenen Bakterien verfolgen.

Auch in dem Rückenmark sind die Influenzabakterien in trockenen Präparaten und in Schnitten zu sehen. Sie scheinen vom Canalis centralis aus in die graue Substanz sich auszubreiten. Man sieht die Epithelialschicht der Canalwandungen zerstört, die Zellen sind mit Bakterien vollgepfropft; eine grosse Einwanderung in die graue Substanz ist aber nicht nachweisbar. Ausserdem zeigten sich auch in Medullarschnitten zahlreiche hämorrhagische Punkte, zahlreiche Leukocjten waren auch bemerkbar, obwohl uicht in so ausgeprägter Weise wie im Gehirn. Bakterien waren bis zur Cauda equina nachweisbar.

Um nun zu sehen, ob das beobachtete Krankheitsbild und der Obductionsbefund als specifisch nur von den Influenzabakterien hervorgerufen zu betrachten sind, oder ob auch andere Bakterien im Stande sind ähnliche Erscheinungen zu machen, waren Controlversuche mit letzteren nothwendig. Ich habe deswegen diese Controlversuche recht zahlreich angestellt, sowohl einmal mit nichtpathogenen Keimen, des Weiteren mit mässig pathogenem Impfmaterial und endlich mit virulenten Bakterien. 
a) Versuche mit nichtpathogenen Keimen.

Mit drei nichtpathogenen Bakterien, die ich eben zur Hand hatte, mit einer gelben Sarcine, mit einem aus Wasser gezüchteten Vibrio und mit einem aus der Luft gewonnenen Staphylococcus - die letzten zwei wuchsen sehr üppig bei $37^{\circ} \mathrm{C}$., die erste sehr spärlich - habe ich nun meine Versuche angestellt, die ich eben vorführe.

Kaninehen 54. $1650^{\mathrm{grm}}$. 1 Normalöse einer, Agarcultur von Sarcine ins Gehirn. Erfolg: Das Thier bleibt am Leben ohne irgend welche krankhafte Erscheinungen zu haben.

Kaninchen $55.1420 \mathrm{grm}$. Controle. 1 Normalöse desgl. intraperitoneal. Erfolg: Bleibt am Leben.

Kaninchen 53. $1580^{\mathrm{grm}}$. 1 Normalöse einer Agareultur von Staphylococcus ins Gehirn. Erfolg: Bleibt am Leben.

Kaninchen 126. $1590 \mathrm{grm}$. 3 Normalösen desgl. ins Gehirn. Erfolg: Bleibt am Leben.

Kaninchen 127. $1470^{\mathrm{grm}}$. Controle. 3 Normalösen Staphylococeus subcutan. Erfolg: Kein Abscess.

Kaninchen 128. $1680^{\mathrm{grm}}$. Controle. 3 Normalösen Staphylococcus intraperitoneal. Erfolg: Bleibt am Leben.

Kaninehen 78. $1760 \mathrm{grm}$. 1 Normalöse einer Agarcultur von Vibrio ins Gehirn. Erfolg: Keine krankhafte Erscheinungen. Bleibt am Leben.

Kaninchen 79. $1520 \mathrm{grm}$. 1 Normalöse desgl. intraperitoneal. Erfolg: Bleibt gesund.

Wie aus diesen Experimenten hervorgeht, sind nicht alle lebende Keime, auch in grösseren Dosen ins Gehirn übertragen, im Stande Erkrankungen mit Tod der Versuchsthiere in ähnlicher Weise zu verursachen wie der Influenzabacillus.

b) Versuche mit mässigpathogenen Bakterien.

Andere Bakterien, die nur eine minimale Pathogenität für Kaninchen haben, waren, ins Kaninchengehirn geimpft, für diese Thiere pathogen. So erlagen die Thiere an der bekannten seit Jahren im Institut für Infectionskrankheiten auf künstlichem Nährboden fortgezüchteten Cholera von Calcutta, obwohl diese Cultur bereits lange ihre Fähigkeit Thiere durch Infection von der Bauchböhle oder vom Subcutangewebe aus zu tödten verloren hatten.

Auch ein Diplococcus, welchen ich von einer Bartolinitis gezüchtet hatte, und der für Mäuse pathogen, für Kaninchen aber nahezu unwirksam 
war, vermochte bei Kaninchen, in's Gehirn geimpft, Erkrankung und Tod dieser Thiere an Septicämie hervorzurufen.

Kaninchen 137. 1520 grm. 3 Normalösen Calcuttacholera ins Gehirn. Erfolg: Stirbt in 18 bis 20 Stunden. Der Sectionsbefund ergab eine starke Entzündung der Meningen und des Gehirns mit zahlreichen Cholerabakterien in Reinculturen. Hyperämie aller Organe war vorhanden.

Kaninchen 145. 3 Normalösen Calcuttacholera in die Bauchhöhle. Erfolg: Das Thier bleibt gesund.

Kaninchen 144. $1670^{\mathrm{grm}}$. Eine Cultur Diplococcus $G$ subcutan. Erfolg: Kein Abscess, keine Infiltration; das Thier bleibt am Leben.

Kaninchen 153. $1420^{\mathrm{grm}}$. 5 Oesen einer in $1^{\mathrm{ccm}}$ Bouillon aufgeschwemmten Cultur des Diplococcus $G$ ins Gehirn. Erfolg: Das Thier ist nach 18 Stunden gestorben. Im Blute ist mikroskopisch und culturell eine starke Septicämie bemerkbar. Die Meningen sind stark hyperämisch mit blutigserösem Exsudat. Das Gehirn ist stark hyperämisch und voll von Diplokokken.

Kaninchen 154. $1610^{\mathrm{grm}}$. Controle. 5 Oesen einer in $1 \mathrm{~cm}$ Bouillon aufgeschwemmten Cultur von Diplokokken in die Bauchhöhle. Erfolg: Bleibt gesund.

c) Versuche mit für Kaninchen pathogenen Bakterien.

Cholera, Coli, Pyocyaneus, Staphylococcus aureus, Typhus zeigten ins Gehirn geimpft eine sehr grosse Pathogenität; ich habe für diese Experimente die durchschnittlich minimale tödtliche Dosis der Influenza angewendet, d. h. $1 / 2 \mathrm{mg}$, da ich einen Vergleich zwischen den letzteren und den anderen Bakterien anstellen wollte. Fränkel'sche Diplokokken, Diphtherie, Tetanus habe ich in einer geringeren Dosis in's Gehirn geimpft, da bekanntlich diese Bakterien eine höchst grosse Giftwirkung besitzen.

Kaninchen 34. $2560^{\mathrm{grm}}$. $1 / 2{ }^{\mathrm{ccm}}$ Bouillon mit $1 / 2{ }^{\mathrm{mg}}$ Cholera in's Gehirn. Erfolg: Gestorben in 20 Stunden.

Kaninchen 35. $2100^{\mathrm{grm}}$. Controle. $2^{\mathrm{mg}}$ Cholera in die Bauchhöhle. Erfolg: Am Leben geblieben.

Kaninchen 314. $1650^{\mathrm{grm}} .1 / 2 \mathrm{~cm}$ mit $1 / 2 \mathrm{mg}$ Coli in's Gehirn. Erfolg: Gestorben in 20 Stunden.

Kaninchen 39. $1425^{\mathrm{grm}}$. Controle. $2^{\mathrm{mg}}$ Coli intraperitoneal. Erfolg: Am Leben geblieben.

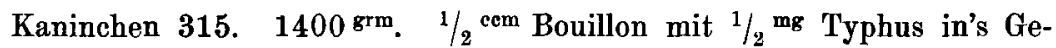
hirn. Erfolg: Gestorben in 20 Stunden.

Kaninchen 51. $1260 \mathrm{grm}$. Controle. $1 / 2^{\mathrm{cem}}$ mit $2^{\mathrm{mg}}$ Typhus intraperitoneal. Erfolg: Am Leben geblieben. 
Kaniuclien 317. $1750^{\mathrm{krm}}$. 1/2 ${ }^{\mathrm{em}}$ mit $1 / 2 \mathrm{mg}$ Staphylococcus aureus in's Gehirn. Erfolg: Stirbt nach ungefähr 20 Stundell.

Kaninchen 56. $1830^{\mathrm{grm}}$. Controle. $2^{\mathrm{mg}}$ Staphylococcus in die Bauchhöhle. Erfolg: Stirbt nach 48 Stunden an eitriger Peritonitis.

Kaninchen 316. $1650 \mathrm{grm}$. $1 / 2{ }^{\mathrm{cm}}$ mit $1 / 2$ mg Pyocyaneus in's Gehirn. Erfolg: Stirbt nach 20 Stunden.

Kaninchen 66. $1720 \mathrm{grm} . \quad 2$ my Pyocyaneus in die Bauchhöhle. Erfoig: Bieibt an Leben.

Kaninchen 131. 2320 grm. 1 Oese einer in $1^{\text {ecm }}$ Bouillon aufgeschwommter Cultur eines Fränkel'schen Diplococcus. Erfolg: Am nächsten Tage allgemeine Paralyse, leichte Krämpfe. Stirbt nach 48 Stunden. Allycemeine Septicämie, sehr starke Meningitis und Encephalytis, massenhaft Diplokokken im Gehirn.

Kaninchen 139. $1690 \mathrm{grm}$. Controle. 1 Oese derselben Aufsebwemmung intraperitoneal, Erfolg: Das Thier bleibt am Leben.

Kaninchen 155. 2300 grm. Controle. 1 Oese derselben Aufschwemmung in die linke Ohrvene. Erfolg: Nach 4 Tagen gallertartiges Oedem am Orte der Injection. Stirbt nach 8 Tagen mit Septicämie.

Der Fränkel'sche Diplococeus besitzt also eine schr grosse Pathogenität in's Gehirn geimpft.

Kaninchen 208. $1680^{\mathrm{grm}}$. 1/3 Normalöse einer 24 stündigen Agarcultur von Diphtherie in's Gehirn. Erfolg: Am letzten Tage leichte Paralyse der Hinterbeine. Nach zwei Tagen totale Paralyse und Dyspnoë. Das Thier stirbt nach 3 Tagen. In den Meningen sind enorme Hämorrhagieen. und blutigseröses Exsudat bemerkbar. Im Gehirne nichts Auffallendes; es konnten keine Bakterien aus dem Gehirn gezüchtet werden. Peritonitis, Nephritis, Milz vergrössert, Nierenkapsel hyperämisch. Leber in incipienter Verfettung.

Kaninchen 233. $1750 \mathrm{grm}$. 1/3 Normalöse Diphtherie subcutan. Erfols: Es tritt ziemlich starke Infiltration an der Stelle der Impfungr auf; nach 9 Tagen ist las Thier wieder gesund und das Gewicht, welches herabgesunken war, nimmit wieder zu. Nach 20 Tagen ist das Thier noch immer am Leben. Gewicht $1800^{\mathrm{grm}}$.

Kaninchen 167. 2320 grm. 1 Oese einer 4 tägigen Tetanusbouilloncultur in's Gehirn. Erfolg: Nach 8 Tagen lebendig und gesund.

Kaninchen 197. $1820 \mathrm{grm}, 4$ Oesen derselben Cultur in's Gehirn. Enfolg: Das Thier bieibt am Leben.

Kaninchen 200. $1750 \mathrm{crm}$. 1/2 $\mathrm{cm}$ derselben Cultur in's Gehirn. Erfolg: Am nächsten Tage etwas Dyspnoë, das Thier läuft wie verrückt herum, stïsst gegen die Wände; uach 23 Stunden bekommt es sehr starke Krämpfe mit tetanischen Contractionen und stirbt wenige Minuten später. Der Scetionshefund war der einer allgemeinen Vergiftung. Die Meningen waren stark geröthet, verdiekt und enthiclten blutigserüses Exsudat; im Gehirn 
nicht Auffallendes; es ist mir nicht gelungen aus dem Gehirn wieder Tetanus zu züchten.

Kaninchen 201. $1670^{\mathrm{grm}}$. Controle. $1 / 2 \mathrm{~cm}$ derselben Cultur subcutan. Erfolg: Keine krankhafte Erscheinungen. Bleibt am Leben.

Maus 165. 2 Oesen derselben Cultur subcutan. Erfolg: Nach 24 Stunden bekommt sie Tetanussymptome; nach 36 Stunden tritt der Tod ein.

In allen diesen Versuchen ist die Pathogenität der genannten Bakterien eine entschieden grössere als nach Injection ins Unterhautbindegewebe oder in die Bauchhöhle; mithin bildet das Gehirn entschieden ein Locus minoris resistentiae. Diese Eingangspforte wird aber bei spontaner Infection nur von wenigen Bakterienarten benutzt. Unter natürlichen Verhältnissen muss die Infection von der Nase oder vom Ohr aus ihren Weg nehmen. Dieser Weg wird nur von den pathogenen Kokken und Influenzabacillen beschritten. Aus diesem Grunde haben die mit Cholera und anderen Bakterien erzeugten Zustände nur ein theoretisches Interesse, da sie bei spontaner Infection im Gehirn nicht gefunden werden.

Nachdem wir so gefunden haben, dass das Gehirn des Kaninchens ein günstiges Nährsubstrat für Influenzabakterien bildet, von wo aus sie in relativ kleinen Dosen eine tödtliche Infection hervorzurufen im Stande sind, erschien es nicht uninteressant zu erfahren, ob durch längeres Fortzüchten in diesem günstigen Nährsubstrat sich auch eine Steigerung der Virulenz bemerkbar machte; um dieses experimentell festzustellen sind die folgenden Versuche angestellt.

Ich ging aus von einer sehr wenig virulenten Cultur IB. M., die ich der Liebenswürdigkeit meines Collegen Dr. Delius verdanke; die Virulenz von dieser Cultur habe ich in den folgenden Experimenten an Meerschweinchen und Kaninchen festgestellt.

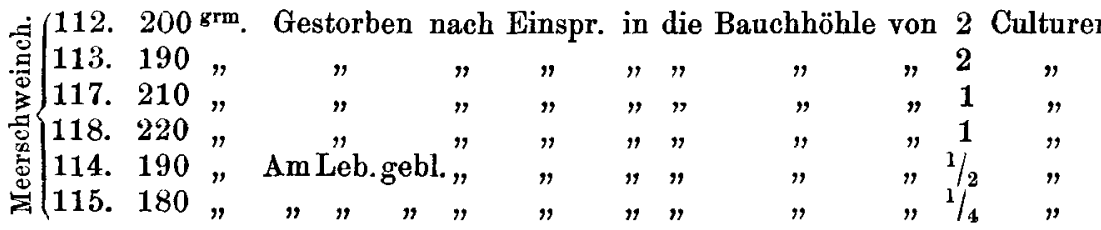

Die minimaltödtliche Dosis ist also für Meerschweinchen 1 Cultur $=6^{\mathrm{mg}}$.

Bei Kaninchen, die in's Gehirn eingespritzt wurden, tödtete erst eine Dosis von $1 / 2$ Cultur $=\mathbf{3}^{\mathbf{m g}}$.

Kaninchen 119. $1820^{\mathrm{grm}}$. Gestorben nach Einspritzung von $1 / 2$ Cultur in's Gehirn. 
Kaninchen 120. $1750^{\mathrm{grm}}$. Am Leben geblieben nach Einspritzung von $1 / 4$ Cultur in's Gehirn.

Diese Cultur IB. M. wurde nun einem Kaninchen in's Gehirn eingespritzt. Als das Thier starb, wurde sein Gehirn steril aus dem Schädel entnommen und von einem Drittel desselben wurde eine Emulsion in $10 \mathrm{~cm}$ Bouillon gemacht und davon $1 / 2 \mathrm{~cm}$ einem anderen Thiere wiederum ins Gehirn injicirt. Es wurde selbstverständlich immer sorgfältig durch Blutagar und Controlröhrchen geprüft, dass es sich um reine Influenza handelte. In dieser Weise wurden 5 Passagen ausgeführt, und nachher wurde wieder die Virulenz der Cultur auf Meerschweinchen und Kaninchen geprüft, wie aus der folgenden Tabelle hervorgeht.

Kaninchen 152. 1 Cultur IB. M. in's Gehirn. Erfolg: Stirbt nach 20 Stunden.

Kaninchen 158. 1.Cultur aus dem Gehirn von Kaninchen 152. Erfolg: Gestorben.

Kaninchen 159. 1/2 ccm Emulsion vom Gehirn des Kan. 158. Erfolg: †

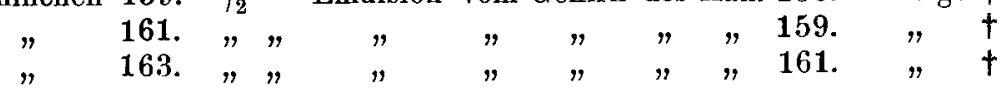

Die Cultur IB. 163 wurde nun auf's Neue auf ihre Virulenz geprüft.

Gestorben sind die Meerschweinchen:

225. $220 \mathrm{grm}$. Mit $6 \mathrm{mg}=1$ Cult. in die Bauchhöhle geimpft,

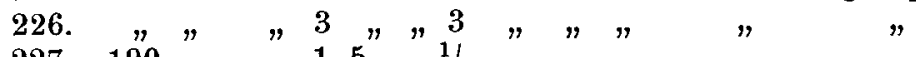

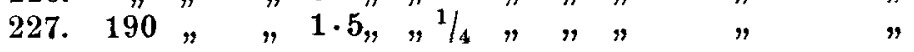

Am Leben geblieben ist Meerschweinchen:

228. . $220^{\mathrm{grm}}$. Mit $0.75^{\mathrm{mg}}=1 / 8$ Cultur in die Bauchhöhle geimpft.

Die minimale für Meerschweinchen tödtliche Dosis, die früher eine Cultur $=6 \mathrm{mg}$ betrug, ist jetzt auf $1 / 4$ Cultur $=1.5^{\mathrm{mg}}$ herabgesunken.

Die Virulenzprüfungen an Kaninchen haben folgendes ergeben:

Kaninchen 212. Bekommt in's Gehirn $4 \mathrm{mg}$. Gestorben in $18 \mathrm{Std}$.

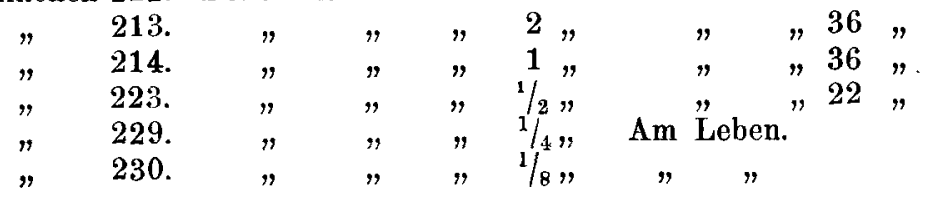

Vorher war die minimaltödtliche Dosis $3^{\mathrm{mg}}=1 / 2$ Cultur, nach fünf Passagen beträgt sie nur $1 / 2 \mathrm{mg}=1 / 12$ Cultur.

Bei einer anderen Cultur, die IB. 176, habe ich schon nach einer Passage eine Erhöhung der Virulenz bemerkt. 
Meerschw. 238. In die Bauchhöhle $18^{\mathrm{mg}}=3$ Culturen IB. 176 . Stirbt.

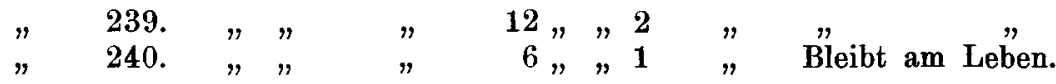

Die Cultur wird nun ins Gehirn eines Kaninchens eingespritzt, das Thier geht nach 18. Stunden ein, und die Culturen, welche aus seinem Gehirne gezüchtet werden, besitzen für Meerschweinchen folgende Virulenz:

Meerschweinchen 252. $244 \mathrm{grm}$. In die Bauchhöhle $5 \mathrm{mg}$. Stirbt nach $18 \mathrm{Std}$. 253. $220, ", " \quad$ " $"$ Bleibt am Leben.

Man sieht also eine ziemliche Erhöhung der Virulenz. Die Dosis letalis minima betrug vor der Einspritzung in's Gehirn des Kaninchens $12 \mathrm{mg}$; nach einer Passage ist sie auf $5^{\mathrm{mg}}$ herabgesunken. Bemerkenswerth ist, dass durch diese Kaninchenpassage auch gleichzeitig eine Virulenzerhöhung für Meerschweinchen stattfindet. Weitere Experimente in diesem Sinne wären sehr wünschenswerth, da es, wie bekannt, gerade mit Influenza sehr schwierig ist, eine virulente Cultur zu haben, oder auf anderem Wege eine Erhöhung der Virulenz zu erlangen. Aber es hat mich oft in diesen Experimenten eine Kaninchenseuche gestört, die zur Zeit unter den Thieren herrschte, und es sehr schwierig machte, viele Passagen durch Thiere auszuführen.

Durch diese Resultate ermuthigt, habe ich durch directe Einspritzungen der Gehirnemulsion eines an Influenza gestorbenen Thieres sehen wollen ob die Kaninchen, die für Influenza soviel wie nicht empfänglich sind, Störungen davon trugen. Es ist mir auch in den meisten Fällen gelungen den Tod hervorzurufen.

Kaninchen 257. $3^{\mathrm{cm}}$ Gehirnemulsion vom an Influenza gestorbenen Kaninchen 246 in die Bauchhöhle. Erfolg: Am näohsten Tag ist das Thier matt und fiebert. Stirbt nach 3 Tagen. Der Sectionsbefund ergab Folgendes: Blutigseröses Exsudat in der Bauchhöhle mit Blutcoagula, Peritonealgefässe stark angefüllt, Milz stark hyperämisch und vergrössert, Leber stark hyperämisch. Die beiden Pleuren sind voll von einem weissgelben Eiter, welcher auch das Pericard erfüllt hat; die rechte Lunge ist vollkommen zerstört, die linke stark hyperämisch. Der Eiter, das peritoneale Exsudat, mikroskopisch und culturell untersucht, enthielten massenhaft Influenzabacillen in Reincultur. Die Controlröhrchen blieben steril.

Kaninchen 262. $3^{\mathrm{ccm}}$ Emulsion Gehirn 261 subcutan. Erfolg: Es bildet sich ein Abscess, welcher, nach 8 Tagen geöffnet, gelblichen Eiter ergiebt; auch von hier konnte man Influenza in Reinculturen züchten. Die mit einfachem Agar geimpften Controlröhrchen blieben steril.

Kaninchen 334. 5 com Emulsion Gehirn 329 in die Bauchhöhle. Nach 3 Tagen ist das Thier gestorben. Der Sectionsbefund ergab eine stark ausgeprägte Peritonitis mit blutigserösem Exsudat, welches Influenzabakterien in Reincultur enthielt; auch hier blieben die geimpften Controlröhrchen steril. 
Kaninchen 335. 5 cem Emulsion Gehirn 329 subcutan. Erfolg: Es bildete sich ein Abscess, welcher, nach 8 Tagen geöffnet, durch Influenzabakterien erzeugten Eiter ergab.

Es ist also die Möglichkeit vorhanden, dass Influenza, auf Kaninchen in dieser Weise geimpft, sich sehr stark pathogen zeigen kann. Die zahlreichen Controlversuche, die ich durch Impfungen von reinen Culturen in die Trachea, in die Brust und Bauchhöhle und subcutan angestellt habe, sind negativ ausgefallen, obwohi ich nicht weniger als 2 bis 3 Culturen den Thieren einimpfte. Um mich nun ein wenig $\mathrm{z}, \mathrm{u}$ orientiren, wodurch die mannigfaltigen Erscheinungen der Influenzabakterien, die sonst so wenig pathogen sind, in den Kaninchen herrorgebracbt waren, ob durch die höhere Virulenz der Gehirnemulsion eines in's Gehirn gespritzten Thieres oder dadurch, dass sie in der Gehirnsubstanz, die mithin eingespritzt wird, einen günstigen Nährboden im Körper der geimpften Thiere fanden, habe ich noch drei Controlversuche angestellt, in welchen ich den Kaninchen eine Emulsion eines normalen Gehirns und Influenzaculturen zusammen einimpfte.

Kaninchen 330. $5 \mathrm{~cm}$ Normalgehirnemulsion und 2 Influenzaculturen subcutan. Erfolg: Das Thier bekommt eined Abscess, der, nach 8 Tagen aufgeschnitten, Eiter mit Influenzabakterien enthätl.

Kaninchen 324. Controle. 3 Culturen subcutan. Erfolg: Keine Infiltration. Bleibt am Leben.

Kaninchen 332. 5 cem Normalgehirnemulsion und 2 Influenzaculturen in die Bauchhöhle. Erfolg: Grestorben nach 2 Tagen mit einer sehr ausgeprägten Peritonitis. Im blutigserösen Exsudat sind Influenzabakterien mikroskopisch und in Reincultur nachgewiesen worden.

Kaninchen 323. Controle. 2 Culturen Influenza in die Bauchhöhle. Erfolg: Völlig gesund.

Es ist also mit grosser Wahrscheinlichkeit anzunehmen, dass die Influenzabakterien dadurch so pathogen wirken, wenn sie mit einer Gehirnemulsion den Kaninchen eingespritzt werden, dass sie in der Gehirnsubstanz einen höchst günstigen Nährboden finden.

\section{Ueber die Wirkung des Influenzagiftes.}

Die bei den intracraniellen Infectionen mit Influenzabakterien am Kaninchenorganismus beobachteten Veränderungen liessen darauf schliessen, dass die Wirkungsweise dieses Bakteriums in der Hauptsache auf eine durch das Gift der Bakterien hervorgerufene Intoxication zurückzuführen ist. Aus den Untersuchungen Pfeiffer's wissen wir schon, dass das Influenzagift ein intracelluläres, d. h. an den Zellenleib gebundenes ist. 
Die Giftmenge steigt und fällt mit der Menge. der injicirten Giftzellen. Bereits Pfeiffer ist es gelungen, die Thiere mit dem Influenzagift von der Blutbahn aus zu tödten, allerdings waren die angewandten Dosen recht hohe. Da wir nun in unseren obigen Experimenten den Nachiveis erbringen konnten, dass das Gift in erster Linie auf das Centralnervensystem einwirkt, so schien am naheliegendsten $\mathrm{zu}$ sein, die abgetödteten Influenzabakterien direct in's Gehirn zu injiciren.

Influenzabakterien wurden in der Weise möglichst schonend abgetödtet, dass sie $1 \frac{1}{2}$ Stunden im Thermostaten bei $57^{\circ} \mathrm{C}$. gehalten wurden. Hierdurch wurde eine sichere Abtödtung aller Keime erzieit. Um ein Kaninchen im Gewicht von 1500 bis $2000^{\mathrm{grm}}$ mit abgestorbenen Influenzabacillen vom Gehirn aus zu tödten, waren bei verschiedenen Influenzastämmen 2 bis $6^{\mathrm{mg}}$ Bakterienmenge nothwendig. Die Zahl schwankt etwas in den einzelnen Versuchen; es mag dieses einmal bedingt sein dureh die verschiedene individuelle Empfänglichkeit der Thiere, andererseits ist dieses Factum auch abhängig vơn der jeweiligen schnelleren oder langsameren Resorption der giftigen Substanzen.

Wurden die Thiere mit tödtlichen Giftmengen subdural geimpft, so stieg die Temperatur schon nach 2 Stunden um einen Grad, erreichte nach 10 bis 12 Stunden ihren Hïhepunkt, oft mit $42^{\circ}$, sank dann allmählich unter die Norm bis zum Tode des Thieres. War die Dosis nicht tödtlich, so zeigte sich ein durch 2 Tage anhaltender fieberhafter Zustand, wobei die Temperatur um 1 bis $2^{0}$ erhöht war. Nach dieser Zeit kehrte sie zur Norm zurück. Die äusseren Erscheinungen entsprechen ganz denen, die wir nach Infection mit lebenden Influenzabakterien beobachtet haben. Besonders auffallend war das Hervortreten der paralytischen Erscheinungen, welche in der Regel zum Tode führen. Hin und wieder kam es indess vor, dass ein Thier selbst nach völligster Lähmung des ganzen Körpers sich noch wieder erholen konnte. Das Zurückgehen der Erscheinungen ist indess ein äusserst langsames. Dabei magern die Thiere ganz enorm und rapide $a b$, so dass beispielsweise bei einem Thiere, welches im Laufe von 2 Monaten dreimal mit je einem Drittel Cultur eingespritzt wurde, das Gewicht von 2115 auf $1040^{\text {grm }}$ herabsank. Dabei kounten wir die Beobachtung machen, dass bei jeder Wiederholung der Intoxication die Empfänglichkeit des Thieres für das Gift eine jedes Mal ganz bedeutend gesteigertere war, so dass von einer Giftfestigkeit hier wohl keine Rede sein kann.

Bei der Obduction der der Vergiftung erlegenen Thiere finden wir in der Bauchhöhle fast immer ein blutig seröses Exsudat; gleichzeitig beobachten wir eine leichte Hyperämie der Nebennieren, starke Hyperämie der Leber mit entzündlicher Veränderung des Parenchyms dieser Organe. 
Die Meningen sind hyperämisch, in der Gehirnsubstanz finden sich hin und wieder kleine Hämorrhagieen.

Zur Illustration des eben Gesagten mögen folgende Thierversuche dienen:

Kaninchen 42, $1670^{\mathrm{grm}}$. In's Gehirn $3^{\mathrm{mg}}=1 / 2$ abgetödtete Cultur. Erfolg: Am nächsten Tage Dyspnoë, Paralyse der Hinterbeine. Stirbt nach 36 Stunden.

Kaninchen 18. $1730^{\mathrm{grm}}$. In's Gehirn $3^{\mathrm{mg}}=1 / 2$ abgetödtete Cultur. Erfolg: Starke clonische Krämpfe, Paralyse der Hinterbeine, dann totale Paralyse. Tod nach 24 Stunden.

Kaninchen 68. $2120^{\mathrm{grm}}$. In's Gehirn $3^{\mathrm{mg}}=1 / 2$ abgetödtete Cultur. Erfolg: Dyspnoë, Krämpfe, Paralyse. Stirbt nach 3 Tagen.

Kaninchen 22. $1580^{\mathrm{grm}}$. In's Gehirn $3^{\mathrm{mg}}=1 / 2$ abgetödtete Cultur. Erfolg: Stirbt nach 24 Stunden mit paralytischen Erscheinungen und Krämpfen.

Kaninchen 109. $2130^{\mathrm{grm}}$. In's Gehirn $3^{\mathrm{mg}}=1 / 2$ abgetödtete Cultur. Erfolg: Stirbt nach 24 Stunden.

Kaninchen 249. $1750^{\mathrm{grm}}$. In's Gehirn $3^{\mathrm{mg}}=1 / 2$ abgetödtete Cultur. Erfolg: Paralyse der Hinterbeine. Bleibt am Leben.

Kaninehen 179. $1650^{\mathrm{grm}} .5^{\mathrm{mg}}$ abgetödtete IB. Erfolg: Gestorben nach 24 Stunden.

Kaninchen 284. $1720^{\mathrm{grm}}$. $6^{\mathrm{mg}}=1$ abgetödtete Cultur. Erfolg: Gestorben nach 20 Stunden.

Kaninchen 248. $1840^{\mathrm{grm}}$. $6^{\mathrm{mg}}=1$ abgetödtete Cultur. Erfolg: Gestorben in der Nacht. storben.

Kaninchen 178. $1810^{\mathrm{grm}}$. $7.5 \mathrm{mg}$ IB. Erfolg: In der Nacht ge-

Kaninchen 180 mit $2^{1 / 2}$ mg abgetödtete Cultur. in's Gehirn geimpft,

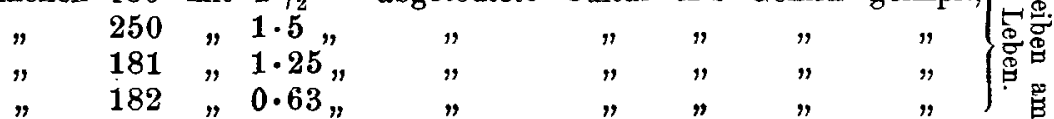

Diese Experimente lehren uns, dass der durch die intracranielle Infection der Kaninchen mit Influenzabakterien hervorgerufene Tod durch die Vergiftung mit dem Gifte dieser Bakterien hervorgerufen wird.

Bei der Infection mit lebendem Materiale kann der Tod nur dann eintreten, wenn die Vermehrung der Giftzellen eine derartige ist, dass sie mindestens $3-6^{\mathrm{mg}}$ ăquivalent sind, entsprechend der tödtlich wirkenden Dosis abgetödteter Culturen.

Da wir aber schon mit minimalsten Mengen lebender Bakterien die Thiere tödten können, so muss im Gehirn der Kaninchen eine lebhafte Vermehrung der Influenzakeime stattgefunden haben. 
Da das Influenzabakteriengift eine solch ausserordentlich toxische Wirkung auf das Centralnervensystem entfaltet, so war es interessant zur Forschung, ob diese Eigenschaft nur dem Influenzagifte eigenthümlich ist oder ob auch die Gifte anderer Bakterienarten, ins Gehirn gebracht, ähnliche Symptomencomplexe auszulösen vermögen. Die in dieser Richtung angestellten Versuche will ich jetzt anführen.

Kaninchen 318. $1500^{\mathrm{grm}}$. $1 / 2{ }^{\mathrm{ccm}}$ Bouillon mit 6 "gg Cholera 3 Stunden bei $57^{\circ}$. Am Leben geblieben. Keine krankhaften Erscheinungen.

Kaninchen 36. $2725^{\mathrm{grm}}$. $1 / \mathrm{c}^{\mathrm{ccm}}$ Bouillon mit $10^{\mathrm{mg}}$ Cholera 3 Stunden bei $57^{0}$. Am nächsten Tage leichte Paralyse der Hinterbeine. Bleibt am Leben. Nach 24 Stunden völlig gesund.

Kaninchen 44. $1930^{\mathrm{grm}}$. $1 / 2{ }^{\mathrm{eem}}$ Bouillon mit $12 \mathrm{mg}$ Cholera 3 Stunden bei $57^{\circ}$. Keine Erscheinungen. Bleibt am Leben.

Kaninchen 319. $1450 \mathrm{grm}$. $1 / 2{ }^{\text {ecm }}$ Bouillon mit $6^{\mathrm{mg}}$ Coli 3 Stunden bei $57^{\circ}$. Ganz gesund, Keine nervösen Erscheinungen.

Kaninchen 320. $1600^{\mathrm{grm}}$. $1 / 2{ }^{\text {com }}$ Bouillon mit $6^{\mathrm{mg}}$ Pyocyaneus 3 Std. bei $57^{\circ}$. Ganz gesund.

Kaninchen 321. $1530 \mathrm{grm}$. $1 / 2 \mathrm{~cm}^{\mathrm{cm}}$ Bouillon mit $6{ }^{\mathrm{mg}}$ Typhus 3 Stunden bei $57^{\circ}$. Ganz gesund.

Kaninchen 82. $1720 \mathrm{grm}, 1 / 2^{\mathrm{eem}}$ Bouillon mit $9 \mathrm{mg}$ Typhus 3 Stunden bei $57^{\circ}$. Völlig gesund.

Kaninchen 322. $1720 \mathrm{grm}$. $1 / 2^{\mathrm{ccm}}$ Bouillon mit $6^{\mathrm{mr}}$ Staphyl. aureus 3 Stunden bei $57^{\circ}$. Keine krankhaften Erscheinungen.

Kaninchen 26. $1770 \mathrm{grm} .1 / 2 \mathrm{~cm}$ Bouillon mit $9 \mathrm{mg}$ Staphyloc. aureus 3 Stunden bei $57^{\circ}$. Am nächsten Tage ganz leichte Paralyse der Hinterbeine. Nach 2 Tagen wieder völlig gesund.

Kaninchen 52. $1590 \mathrm{grm}$. ${ }_{1 / 2} \mathrm{com}$ Bouillon mit $9 \mathrm{mg}$ Staphyloc. aureus 3 Stunden bei $57^{\circ}$. Keine krankhaften Erscheinungen.

Wie aus diesen Experimenten hervorgeht, ist es, so weit die von uns untersuchten Bakterien in Betracht kommen, nicht möglich, mit ähnlichen Dosen wie die der Influenzabacillen ähnliche Effecte wie mit dem Influenzagift auszulösen. Die Einwirkung dieses letzteren auf das Centralnervensystem scheint daher eine erhebliche zu sein, welche wenigstens in den kleinen Dosen nur mit der Influenzawirkung erzeugt werden konnte; da, obwohl die Thiere mit grösseren Dosen der anderen Bakterien vergiftet waren, sie auch während den der Impfung folgenden Tagen keinerlei nervöse Störungen gezeigt haben.

Wenn wir das Facit aus unseren gesammten, an 350 Kaninchen angestellten Versuchen ziehen, so ergiebt sich, dass es möglich ist, an Kaninchen eine Infection mit kleinen Dosen lebender Influeuzabakterien 


\section{A. Cantani Jun.: Wirkung deir Influenzabacillen v. $s$. w.}

hervorzurufen, eine Möglichkeit, die bisher noch nicht festgestellt werden konnte. Diese Möglichkeit ist aber nur dann vorhanden, wenn man den Ort als Angriffsstätte der Influenzabakterien benutzt, welcher einen natürlichen Locus minoris resistentiae bildet, das Gehirn. Denn die Influenzabakterien bilden ein intracelluläres Gift, welches in erster Linie auf das Centralnervensystem besonders schädlich einwirkt.

Am Schluss dieser Arbeit sage ich meinem hochrerehrten Lehrer, Hrn. Geheimrath Koch, sowie auch Hrn. Prof. Pfeiffer meinen verbindlichsten Dank für die Anregung und Unterstützung, die mir bei der Ausführung dieser Experimente zu Theil wurde.

\section{Litteratur.}

Pfeiffer, Vorlänfige Mittheilung über den Erreger der Influenza. Deutsche med. Wochenschrift. 1892. Nr. 2.

Derselbe, Die Aetiologie der Influenza. Diese Zeitschrift. 1892. Bd. XIII.

Pfuhl, Bakteriologischer Befund bei schweren Erkrankungen des Centralnervensystems im Verlauf von Influenza. Berliner klin. Wochenschrift. 1892. Nr. 39.

Pribram, Beiträge zur Kenntniss der Influenza. Prager med. Wochenschrift. 1894. Nr. 7.

Leyden, Ueber multiple Neuritis und acute aufsteigende Paralyse nach Influenza. Zeitschrift für klin. Medicin. Bd. XXII.

Hirschmann, Ueber Neuroretinitis nach Influenza. Festschrift aur Feier des 50jähr. Jubiläums des Düsseldorfer Aerztevereins. Wiesbaden 1894.

Lemcke, Die acute Caries und Nekrose des Felsenbeins nach Influenza. Vortrag bei der dritten Versammlung der deutschen otol. Gesellschaft in Bonn. Berl. klin. Wochenschrift. 1894. Nr. 31.

Voges, Beobachtungen und Untersuchungen über Infiuenza und dem Erreger dieser Erkrankung. Ebenda. 1894. Nr. 38. 